\title{
Is intraoperative real-time dosimetry in prostate seed brachytherapy predictive of biochemical outcome?
}

\author{
Daniel Taussky, MD',2, Levon Igidbashian, MD³, David Donath, MD', Dominic Béliveau-Nadeauv, MSc, \\ Renée X. Larouche, MSc', Yanick Hervieux, MScl, Guila Delouya, MD, MSc',2 \\ 'Department of Radiation Oncology, Centre hospitalier de l'Université de Montréal, Hôpital Notre-Dame, Montréal, QC, ${ }^{2}$ Centre de recherche \\ du Centre Hospitalier de l'Université de Montréal, Montréal, QC, ${ }^{3}$ Department of Radiation Oncology. Centre intégré de santé et de services \\ sociaux Laval, Cité de la Santé, Laval, QC, Canada
}

\begin{abstract}
Purpose: To analyze intraoperative (IO) dosimetry using transrectal ultrasound (TRUS), performed before and after prostate low-dose-rate brachytherapy (LDR-BT), and compare it to dosimetry performed 30 days following the LDR-BT implant (Day 30).

Material and methods: A total of 236 patients underwent prostate LDR-BT using ${ }^{125}$ I that was performed with a three-dimensional TRUS-guided interactive inverse preplanning system (preimplant dosimetry). After the implant procedure, the TRUS was repeated in the operating room, and the dosimetry was recalculated (postimplant dosimetry) and compared to dosimetry on Day 30 computed tomography (CT) scans. Area under curve (AUC) statistics was used for models predictive of dosimetric parameters at Day 30.

Results: The median follow-up for patients without BF was 96 months, the 5-year and 8-year biochemical recurrence (BR)-free rate was $96 \%$ and $90 \%$, respectively. The postimplant median $\mathrm{D}_{90}$ was 3.8 Gy lower (interquartile range [IQR], 12.4-0.9), and the $\mathrm{V}_{100}$ only $1 \%$ less (IQR, 2.9-0.2\%) than the preimplant dosimetry. When comparing the postimplant and the Day 30 dosimetries, the postimplant median $\mathrm{D}_{90}$ was $9.6 \mathrm{~Gy}$ higher (IQR [-] 9.5-30.3 Gy), and the $\mathrm{V}_{100}$ was $3.2 \%$ greater $(0.2-8.9 \%)$ than Day 30 postimplant dosimetry. The variables that best predicted the $\mathrm{D}_{90}$ of Day 30 was the postimplant $\mathrm{D}_{90}(\mathrm{AUC}=0.62, p=0.038)$. None of the analyzed values for IO or Day 30 dosimetry showed any predictive value for $\mathrm{BR}$.

Conclusions: Although improving the IO preimplant and postimplant dosimetry improved dosimetry on Day 30, the BR-free rate was not dependent on any dosimetric parameter. Unpredictable factors such as intraprostatic seed migration and IO factors, prevented the accurate prediction of Day 30 dosimetry.
\end{abstract}

J Contemp Brachytherapy 2017; 9, 4: 304-308 DOI: https://doi.org/10.5114/jcb.2017.68467

Key words: biochemical recurrence, brachytherapy, intraoperative dosimetry, prostate cancer.

\section{Purpose}

In 2001, the American Brachytherapy Society (ABS) provided their critical assessment on intraoperative (IO) planning in prostate permanent seed low-dose-rate brachytherapy (LDR-BT) with ${ }^{125} \mathrm{I}$, and gave recommendation for an online real-time IO dosimetry [1]. In 2010, the Groupe Européen de Curiethérapie and the European Society for Radiotherapy \& Oncology (GEC-ESTRO) group discussed the various ways to use real-time dosimetry and described the basic approaches involving IO planning within the operating room (OR). This consisted of interactive planning with modification of the treatment based on the feedback of the real-time tracking of the actual needle positions and a continuous feedback of the seed positions as they were implanted [2]. In 2008, we described our results with an interactive planning technique using real-time three-dimensional (3D) transrectal ultrasound (TRUS) for dose planning and IO navigation [3], and reported the clinical outcome, toxicity [4,5], and seed loss [6] for low to high activity seeds. We described that IO dosimetric parameters on TRUS could predict a good quality implant on the Day 30 computed tomography $(\mathrm{CT})$ scan. We also discovered that a good IO preimplant plan was the best predictive factor of a good implant on Day 30 [3]. In this present study, we updated our experience with a larger than previously described cohort and investigated whether IO TRUS-based dosimetry was predictive of biochemical recurrence (BR).

Address for correspondence: Daniel Taussky, MD, Department of Radiation Oncology, Centre hospitalier Received: 04.04 .2017 de l'Université de Montréal - Hôpital Notre-Dame, 1560 Sherbrooke St. E., Montreal, Quebec, H2L 4M1 Accepted: 29.05 .2017 Canada, phone: +1 514 890-8254, fax: +1 514 412-7537, e-mail: daniel.taussky.chum@ssss.gouv.qc.ca Published: 30.08 .2017 


\section{Material and methods}

Of the first 251 patients who were treated with LDRBT at our department, complete data were available for 238 (95\%) patients. A postoperative dosimetry was not obtained due to technical difficulties in the other $5 \%$ of patients. In all patients, TRUS (BK Medical Systems, Harlev, Denmark) was performed immediately after the implant of two fixation needles. This TRUS was used for IO pre-planning (preimplant IO dosimetry). The planning and seed delivery were performed using the Nucletron FIRST system (Nucletron, an Elekta company, Elekta AB, Stockholm, Sweden) with IO interactive planning, dynamic dose calculation, virtual needle guidance, robotic seed delivery, and needle retraction system. Immediately after implantation, an additional TRUS study was performed with the fixation needles still in place. Prostate delineation was repeated within the planning system a few days after the implant on the postoperative TRUS images, and the dosimetry to the prostate was recalculated (postimplant IO dosimetry). Slice thickness on both TRUS studies was $2.5 \mathrm{~mm}$.

All patients had CT-based dosimetry performed at 30 days (Day 30) after the implant. The implant technique and dosimetry were performed as previously described [3]. Slice thickness was $3 \mathrm{~mm}$.

\section{Statistical analysis}

Correlations between variables were calculated using the Pearson correlation coefficient. The value of IO dosimetry to predict Day 30 dosimetry was calculated using area under the receiver operating characteristic (AUROC) curves.

Table 1. Patient and dosimetric characteristics $(n=236)$

\begin{tabular}{lc} 
Factor & Median (IQR) \\
\hline Age median & $65(61-69)$ \\
\hline Seed activity $(\mathrm{mCl})$ & $0.59(0.44-0.61)$ \\
\hline Seeds/needles & $2.6(2.4-2.8)$ \\
\hline ADT & $4 \%$ \\
\hline PSA & $5.7(4.4-7.3)$ \\
\hline Gleason score 6/7/8 & $87.5 \% / 12.2 \% / 0.4 \%$ \\
\hline Prostate volume (cc) & $38.6(32-45)$ \\
\hline Preimplant $\mathrm{V}_{100}(\%)$ & $99(98-100)$ \\
\hline Preimplant $\mathrm{D}_{90}(\mathrm{~Gy})$ & $178(173-182)$ \\
\hline Postimplant $\mathrm{V}_{100}(\%)$ & $98(95-99)$ \\
\hline Postimplant $\mathrm{D}_{90}(\mathrm{~Gy})$ & $171(163-177)$ \\
\hline Day $30 \mathrm{~V}_{100}(\%)$ & $93(89-97)$ \\
\hline Day $30 \mathrm{D}_{90}(\mathrm{~Gy})$ & $157(140-177)$
\end{tabular}

$I O R$ - interquartile range, $A D T$ - androgen deprivation therapy, PSA - prostate-specific antigen, $V_{100 \%}$ - volume of the anatomic volume receiving $100 \%$ of the prescribed dose, $D_{90}$ - percent of the prescription dose covering $90 \%$ of the CTV
BR was defined according to the Phoenix definition $($ PSA $=$ nadir $+2 \mathrm{ng} / \mathrm{ml})$. Survival analyses were performed using the Kaplan-Meier method and were compared using the log-rank test.

\section{Results}

Table 1 lists patient and dosimetric characteristics of the 236 patients analyzed. Compared to the preimplant IO dosimetry, the postimplant $\mathrm{D}_{90}$ median was $3.8 \mathrm{~Gy}$ lower (interquartile range [IQR], 12.4-0.9), and the $\mathrm{V}_{100}$ was $1 \%$ less (IQR, 2.9-0.2\%). The postimplant IO $\mathrm{D}_{90}$ was a median of 9.6 Gy higher (IQR, 9.5-30.3 Gy) than on Day 30 , and the postimplant $\mathrm{V}_{100}$ was $3.2 \%$ higher $(0.2-8.9 \%)$ than on Day 30. See Table 2 for differences in dosimetry at the different time-points. The quality of the implant measured with the $D_{90}$ and $V_{100}$ did not change significantly over time. Only the postimplant $\mathrm{V}_{100}$ value increased slightly with the year of implantation $(r=0.19, p=0.004)$, but not the $D_{90}$ without any effect on preimplant or Day 30 values (data not shown).

Figure 1 illustrates the correlation between the preimplant and the postimplant $D_{90}$, and Figure 2 shows the correlation between the postimplant $\mathrm{D}_{90}$ and the $\mathrm{D}_{90}$ on Day 30 as a scatterplot. Both the postimplant IO $\mathrm{D}_{90}$ $(\mathrm{AUC}=0.62, p=0.038)$ and the preimplant $\mathrm{IO} \mathrm{D}_{90}$ (AUC $=0.61, p=0.046)$ were significant predictors of the $\mathrm{D}_{90}$ at Day 30. However, the false positive rate to predict for a $\mathrm{D}_{90} \geq 130$ Gy for different cut-offs was too high to identify ideal cut-offs. The $\mathrm{D}_{90}$ on Day 30 weakly correlated with the postimplant $\mathrm{D}_{90}(r=0.13, p=0.05)$ and with the prostate volume $(r=0.26, p<0.001)$. On Day 30 , the $\mathrm{D}_{90}$ was $<130 \mathrm{~Gy}$ in $13 \%$ of the patients.

Neither the preimplant nor the postimplant $\mathrm{V}_{100}$ showed a clear correlation with the $V_{100}$ on Day 30 . The postimplant $\mathrm{V}_{100}$ had the better predictive ability $(\mathrm{AUC}=0.64, p=0.015)$ for a $\mathrm{V}_{100}$ of $<85 \%(14 \%$ of patients) on Day 30 than the preimplant $\mathrm{V}_{100}(p=0.08)$.

The median follow-up for patients without BR was 96 months (IQR, 66-108). The 5-year and 8-year BR-free rate was $96 \%$ and $90 \%$, respectively. To identify cut-off values predictive of $B R$, we used quartiles of the $D_{90}$ of the preoperative, postoperative, and Day 30 values. None of these quartiles showed any predictive value. This

Table 2. Differences in dosimetry

\begin{tabular}{lcc} 
Factor & Median & IQR \\
\hline $\mathrm{D}_{90}(\mathrm{~Gy}) \Delta$ postimplant - preimplant & -3.8 & -12.4 to -0.9 \\
\hline $\mathrm{D}_{90} \Delta$ postimplant - Day 30 & 9.6 & -9.5 to 30.3 \\
\hline $\mathrm{D}_{90} \Delta$ preimplant - Day 30 & 20.1 & -0.8 to 36.8 \\
\hline $\mathrm{V}_{100}(\%) \Delta$ postimplant - preimplant & -0.95 & -2.9 to -0.2 \\
\hline $\mathrm{V}_{100} \Delta$ postimplant - Day 30 & 3.2 & 0.2 to 8.9 \\
\hline $\mathrm{V}_{100} \Delta$ preimplant - Day 30 & 5.4 & 1.9 to 9.6
\end{tabular}

$1 Q R$ - interquartile range, $\Delta$-difference, $V_{100 \%}$-volume of the anatomic volume receiving $100 \%$ of the prescribed dose, $D_{90}$ - percent of the prescription dose covering $90 \%$ of the CTV 


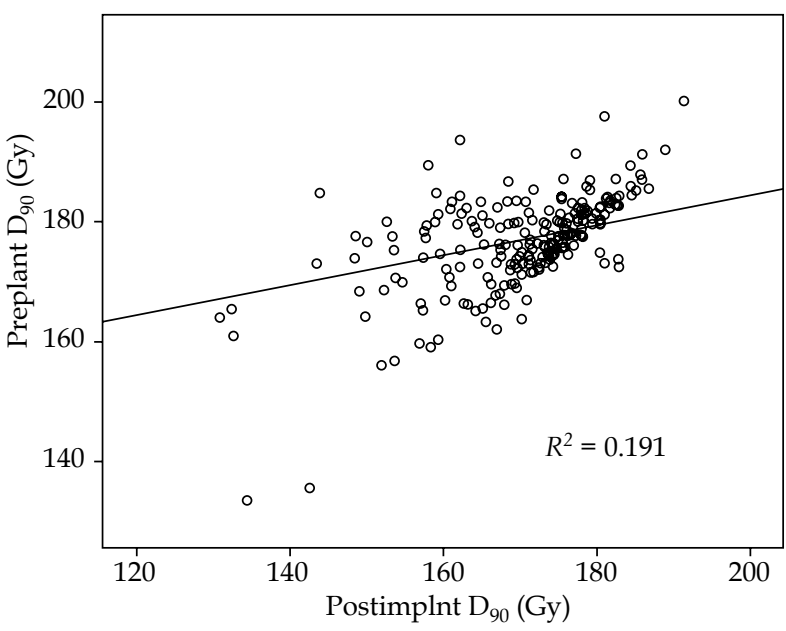

Fig. 1. Correlation between the preimplant D90 and the postimplant D90 as a scatterplot

was also true when applied for a cut-off of 130 Gy for the $\mathrm{D}_{90}$ of Day 30 (detailed results not shown, all $p \geq 0.2$ ). The year of the implant also did not have an influence on BR $(p=0.8)$. To account for a possible learning-curve, we repeated the analysis, this time excluding the first $10 \%$ $(n=23)$ of patients. None of the tested quartiles or the $D_{90}$ on Day 30 was predictive of BR. Neither were such factors as Gleason score, PSA, or PSA-density (results not shown).

\section{Discussion}

We present one of the largest studies on IO planning and dose-delivery for prostate LDR-BT with a long follow-up. We discovered that a high $\mathrm{D}_{90}$ on the preplanning as well as on the postoperative TRUS were predictive of a good ( $\geq 130 \mathrm{~Gy}$ ) $\mathrm{D}_{90}$. In addition, a high $\mathrm{V}_{100}$ on the postoperative TRUS dosimetry was significantly $(p=0.015)$ predictive of a $\mathrm{V}_{100} \geq 85 \%$, although the AUC (0.64) was low, making this measurement an unreliable factor. However, due to the relatively low AUC value $(<0.7)$ of all values and a lack of correlation between the values on Day 30 and the IO values, we determined that the IO dosimetry cannot reliably predict for Day 30 dosimetry. Therefore, Day 30 dosimetry remains the gold-standard in the evaluation of implant quality. In fact, many studies published before about 2010 have shown Day 30 dosimetry to predict for BR [7]. With a median follow-up of 8 years, we sought to identify cut-offs from IO dosimetry to predict BR. The fact that the quartiles, including the worst versus best quartiles of $\mathrm{IO}$ and Day 30, did not predict for BR, is likely due to the small sample size of 238 patients.

Others have described positive results in patients treated with intraoperative planning [8]. Indeed, our results are similar to a recent publication by Shukla et al. [9] using real-time intraoperative planning. They describe an eight-year BR-free rate of $87-92 \%$, comparable to our $90 \%$ and, as in our present study, an absence of any dosimetric parameters predicting BR. Another reason for the lack of predictive value of dosimetry might be that there are

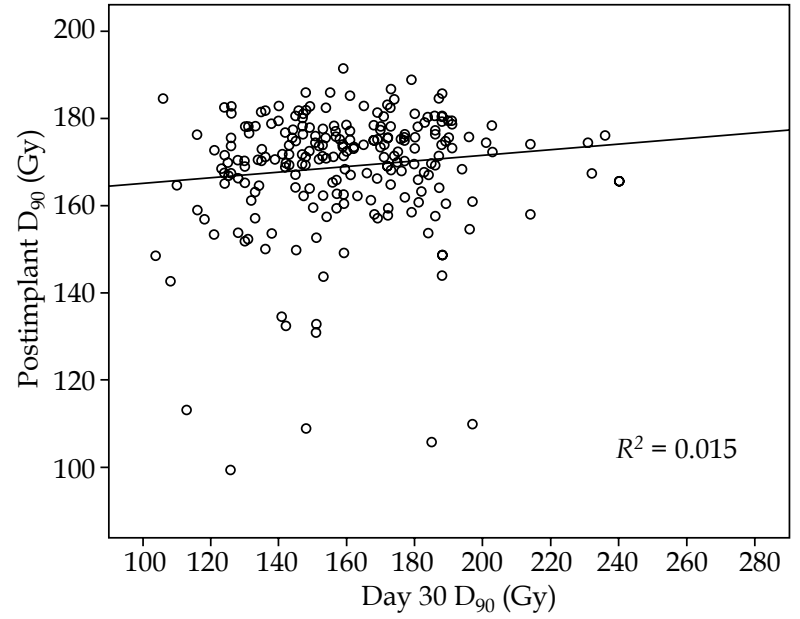

Fig. 2. Correlation between the postimplant D90 and the D90 on Day 30 as a scatterplot

many other factors that may play a role in outcome determination such as classical risk factors for biochemical recurrence including PSA and Gleason score as well as PSA density, which was recently described by our group [10].

Day 30 dosimetry is more accurate and less interobserver-dependent when performed with magnetic resonance imaging (MRI)-CT fusion than with CT alone, and is more accurate for prostate-sector analysis [11,12]. In fact, the use of MRI in the evaluation of postimplant dosimetry has recently been recommended by the American College of Radiology (ACR) [13].

The reasons why dosimetry decreases from preimplant to postimplant, and then to Day 30 are manifold. First, the decrease in dosimetry from preimplant to postimplant is influenced by factors such as non-anticipated pubic-arch interference and prostate swelling during the implant. The decrease to Day 30 is influenced by difficulties during the procedure in correctly identifying the prostate, and probably most importantly, intraprostatic migration after the implant [14] and seeds being displaced by the movement of the needle, or the creation of a vacuum or tunnel in the prostatic tissue by the needle. Another factor influencing dosimetry could be urinary catheter placement [15], but no catheter was placed on Day 30, although catheters are in place intraoperatively. Furthermore, we didn't evaluate interobserver or intraobserver variability in contouring the prostate on TRUS, which is lower than on CT and has a smaller effect on dosimetry. This is due to the better soft-tissue contrast at the prostate border on TRUS $[16,17]$. It is known that very small changes on CT contouring can have a large effect on dosimetry [18].

Unfortunately, with the present software, we find it very difficult to analyze which seeds were displaced and by how much.

Evaluation and comparison of prostate contours before and after the implant are difficult. In our study, the preimplant dosimetry was done after the implantation of 2 fixation needles, which caused significant prostate edema. Although we previously found that the edema caused by the implant compared to the prostate volume after the 
insertion of the fixation needles was small (mean 1.9 cc) [19]; the prostate borders are more difficult to accurately define after the implant because of the edema and other artifacts caused by the procedure. The best way to analyze the reasons for the decline in dosimetry would be to use magnetic resonance imaging (MRI) immediately after the implant as well as on Day 30. Another more feasible option to improve intraoperative prediction of Day 30 dosimetry is the use of intraoperative fusion computed tomography [20].

The major limitation of IO planning with most currently available IO planning systems is the inability to adequately update the delivered seed position [1]. This may explain the absence of a meaningful intraoperative prediction of Day 30 dosimetry and BR in our present study. Other weak points of our study are that there was no IO protocol for the several physicists in the OR. Therefore, planning practices as well as when and how to update the needle position, may have differed between physicists. The treated patients represent the first cases of LDR-BT in our department. Although learning-curve effect on the implant quality and technique is probable, our long-term biochemical results are comparable with the literature. The same physician contoured the prostates on TRUS as well as on CT on Day 30. We therefore did not account for interobserver variability in contouring.

Intraoperative planning techniques were developed to overcome some of the shortfalls of the classic preplanning approach. These shortfalls include difficulties in matching the prostate TRUS image from the preplan to the IO position, adjusting for prostate shape and volume changes, and the additional work and discomfort to the patients caused by preplanning study. As such, IO planning has many advantages. Real-time IO treatment planning software with inverse planning optimization has been shown to achieve excellent implants [21,22]. Several centers have shown better dosimetric results from IO planning, as reviewed by Polo et al. [2]. To our knowledge, this is the first study with an exceptionally long follow-up, analyzing the impact of IO preimplant and postimplant dosimetry on BR.

Intraoperative 3D dose-planning has been shown to reduce the dose to the rectum and urethra, and allows for a reduction in numbers of seeds and $\mathrm{mCi}$ delivered [23]. IO with inverse optimization, as used in our cohort, has also been shown to produce less urethral dose, and a better implant than computer-assisted optimization methods modified peripheral and geometric optimization. Furthermore, it has been shown to result in a higher median $\mathrm{V}_{10}$ and $\mathrm{D}_{90}$ with fewer needles and seeds [24].

\section{Conclusions}

In conclusion, we found that IO dosimetry was predictive of Day 30 dosimetry, but with a low predictive value. We could not identify IO dosimetry that would predict biochemical outcome. We, therefore, conclude that postoperative CT- and MRI-based dosimetry remains the gold standard for evaluation of implant quality [12,25]. The incorporation of functional imaging, MRI-based IO planning, or use of robotics in seed delivery requires further study to determine if they will improve IO planning.

\section{Disclosure}

Authors report no conflict of interest.

\section{References}

1. Nag S, Ciezki JP, Cormack R et al. Intraoperative planning and evaluation of permanent prostate brachytherapy: report of the American Brachytherapy Society. Int J Radiat Oncol Biol Phys 2001; 51: 1422-1430.

2. Polo A, Salembier C, Venselaar J et al. Review of intraoperative imaging and planning techniques in permanent seed prostate brachytherapy. Radiother Oncol 2010; 94: 12-23.

3. Igidbashian L, Donath D, Carrier J-F et al. Poor predictive value of intraoperative real-time dosimetry for prostate seed brachytherapy. Int J Radiat Oncol Biol Phys 2008; 72: 605-609.

4. Tétreault-Laflamme A, Zilli T, Meissner A et al. The Quadrella: A novel approach to analyzing optimal outcomes after permanent seed prostate brachytherapy. Radiother Oncol 2014; 111: 110-113.

5. Zilli T, Taussky D, Donath D et al. Urethra-sparing, intraoperative, real-time planned, permanent-seed prostate brachytherapy: toxicity analysis. Int J Radiat Oncol Biol Phys 2011; 81: e377383.

6. El-Bared N, Sebbaq N, Béliveau-Nadeau D et al. Seed loss in prostate brachytherapy. Strahlenther Onkol 2016; 192: 305-311.

7. Potters L, Roach M 3rd, Davis BJ et al. Postoperative nomogram predicting the 9-year probability of prostate cancer recurrence after permanent prostate brachytherapy using radiation dose as a prognostic variable. Int J Radiat Oncol Biol Phys 2010; 76: 1061-1065.

8. Guinot JL, Ricós JV, Tortajada MI et al. Comparison of permanent 125I seeds implants with two different techniques in 500 cases of prostate cancer. J Contemp Brachytherapy 2015; 7: 258-264.

9. Shukla G, Sarkar A, Hanlon A et al. Biochemical control and toxicity for favorable-and intermediate-risk patients using real-time intraoperative inverse optimization prostate seed implant: Less is more! Brachytherapy 2017; 16: 490-496.

10. Benzaquen D, Delouya G, Ménard C et al. Prostate-specific antigen density is predictive of outcome in suboptimal prostate seed brachytherapy. Brachytherapy 2017; 16: 348-352.

11. Maletz KL, Ennis RD, Ostenson J et al. Comparison of CT and MR-CT fusion for prostate post-implant dosimetry. Int J Radiat Oncol Biol Phys 2012; 82: 1912-1917.

12. Crook J, McLean M, Yeung I et al. MRI-CT fusion to assess postbrachytherapy prostate volume and the effects of prolonged edema on dosimetry following transperineal interstitial permanent prostate brachytherapy. Brachytherapy 2004; 3: 55-60.

13. Davis BJ, Taira AV, Nguyen PL et al. ACR appropriateness criteria permanent source brachytherapy for prostate cancer. Brachytherapy 2016, 16: 266-276.

14. Wang Y, Nasser NJ, Borg J, Saibishkumar EP. Evaluation of the dosimetric impact of loss and displacement of seeds in prostate low-dose-rate brachytherapy. J Contemp Brachytherapy 2015; 7: 203-210.

15. Kunogi H, Yamaguchi N, Wakumoto Y, Sasai K. Effect of a urinary catheter on seed position and rectal and bladder doses in CT-based post-implant dosimetry for prostate cancer brachytherapy. J Contemp Brachytherapy 2015; 7: 211-217.

16. Xue J, Waterman F, Handler J, Gressen E. The effect of interobserver variability on transrectal ultrasonography-based postimplant dosimetry. Brachytherapy 2006; 5: 174-182.

17. Haworth A, Ebert M, Clair SS et al. Impact of selection of post-implant technique on dosimetry parameters for permanent prostate implants. Brachytherapy 2005; 4: 146-153. 
18. Mashouf S, Safigholi H, Merino T et al. Sensitivity of clinically relevant dosimetric parameters to contouring uncertainty in postimplant dosimetry of low-dose-rate prostate permanent seed brachytherapy. Brachytherapy 2016; 15: 774-779.

19. Chira C, Delouya G, Larrivée S et al. Prostate volume changes during permanent seed brachytherapy: an analysis of intra-operative variations, predictive factors and clinical implication. Radiat Oncol 2013; 8: 177.

20. Ishiyama H, Sekiguchi A, Satoh T et al. Dosimetry of permanent interstitial prostate brachytherapy for an interoperative procedure, using O-arm based CT and TRUS. J Contemp Brachytherapy 2016; 8: 7-16.

21. Zelefsky MJ, Yamada Y, Cohen GN et al. Intraoperative real-time planned conformal prostate brachytherapy: post-implantation dosimetric outcome and clinical implications. Radiother Oncol 2007; 84: 185-189.

22. Beaulieu L, Evans DA, Aubin S et al. Bypassing the learning curve in permanent seed implants using state-of-the-art technology. Int J Radiat Oncol Biol Phys 2007; 67: 71-77.

23. Raben A, Chen H, Grebler A et al. Prostate seed implantation using 3D-computer assisted intraoperative planning vs. a standard look-up nomogram: Improved target conformality with reduction in urethral and rectal wall dose. Int J Radiat Oncol Biol Phys 2004; 60: 1631-1638.

24. Raben A, Sammons S, Sim S et al. Initial comparison of inverse optimization, modified peripheral technique, and geometric optimization as real-time intraoperative computer planning options for permanent seed implantation of the prostate. Brachytherapy 2007; 6: 238-245.

25. Polo A, Cattani F, Vavassori A et al. MR and CT image fusion for postimplant analysis in permanent prostate seed implants. Int J Radiat Oncol Biol Phys 2004; 60: 1572-1579. 